\section{A Few Remarkable TEM Facts}

\author{
Phil Fraundorf, University of Missouri
}

What follows is a list of some physical perspectives on the electrons used routinely for transmission electron microscopy. Without knowing it, you may on a daily basis be putting to practical use things, like the wave nature of electrons, that were inconceivable in the early part of this century. In fact, some of the properties of these electrons may be only marginally conceivable today!

Fast Electrons: A back of the envelope calculation for $300 \mathrm{keV}$ electrons gives gamma $=(300=511) / 511=1.587$, so that they travel at $w=$ $c^{2}\left[1-(1 \text {-gamma })^{2}\right]=0.777 c$ or (lightyears per inertial year) of elapsed time. However, if we consider traveler (i.e., electron or proper) time for such a speeding electron, this would give that the travel $u=$ gamma $^{\star} w=1.232$ lightyears per traveler year of elapsed time! With this spatial 4-vector velocity well over $c$, we're dealing with relativity in action! I wonder how many g's of acceleration they experience in the electron gun in order to get up to speed? For more on this subject, you might want to check our browser-interactive relativistic Accel-One problem solver, and the theory pages attached, at http://newton.umsl.edu/-run/index.html

Lonely Electrons: I think that it was John Armstrong at Caltech who once pointed out to me that the number of microscope beam electrons in your TEM specimen at any one time is so small that the odds of such electrons interfering with each other to form diffraction patterns is quite small. The vertical separation between electrons in the column is well, where $/$ is the specimen current, $w$ is the electron inertial velocity, and $e$ is the charge per electron. For an nanoamp of $300 \mathrm{kV}$ electrons, this is $(0.777$ $\left.\times 3 \times 10^{8} \mathrm{~m} / \mathrm{s}\right) \times\left(1.6 \times 10^{-19} \mathrm{C} / \mathrm{e}\right) /\left(10^{-9} \mathrm{C} / \mathrm{s}\right)=0.037 \mathrm{~m} / \mathrm{e}$. Under some illumination conditions there may be no more than 1 beam electron in the column at a time! Hence diffraction patterns in the TEM are basically formed by individual elections interfering with themselves! As you know, such interference will occur only if we don't take steps to determine the path of individual electrons through the specimen! If we look too closely at these paths, the diffraction patterns would disappear (cf. Englert et al., Scientific American, Dec. 1994, 86-92 on quantum erasure).

Fat Electrons: The transverse coherence widths of electrons which make possible electron phase contrast (HREM) lattice imaging and probably electron holography might also be seen as lateral broadening of individual electron wave-packets via the uncertainty principle, which results because we know too much about their transverse momentum! My intuition tells me that we're talking about lateral wave-function spreads of, say, 15 Angstroms in a $\mathrm{LaB}_{6} \mathrm{HREM}$ to more than 100 Angstroms in field emission gun systems. Are these numbers reasonable? By increasing the spread of electron angles in the incident beam, this transwerse coherence width can presumably be decreased (e.g. you want it small for Z-contrast imaging (I think), or varied as in the variable coherence-width strategies of Murray Gibson at U. of I.

Long Electrons: The tight tolerances on high voltage stability and the emitted spread in electron energies means that our uncertainty in the longitudinal momentum of TEM electrons is quite small, and hence again by the uncertainty principle that the wave-packet spread in the direction of motion for TEM electron can be quite large. Distances of, say, 1000 Angstroms come to mind! The associated tight distribution of incident electron energies decreases chromatic and instability damping of fine details in CTEM and HREM images, so that for most applications you may want your electrons "as long as possible". An exception might be in variablecoherence strategies (mentioned above), where shorter electrons might provide sensitivity to shorter-range vertical correletions.

The foregoing thoughts on fast, lonely, fat, and long electrons are not really things I've had time to think much about, but they are interesting, and hence I would enjoy other perspectives on them, as well as suggestions for other "remarkable TEM facts" to add to the list! A "live" draft of this list will be accessible through our scanned Tip \& Electron Image Lab page at: htto://newton.umsl.edu/stel-lab/ 